stresses can become so large that electrons are eventually torn from their atomic orbits and crushed into the nuclei. The result is a closely packed lattice of about $10^{57}$ neutrons, squashed to a nuclear density one-billion times higher than within a white dwarf. A strongly repulsive degeneracy pressure now arises between the neutrons and the squeezing is halted, eventually leaving a 'neutron star' in stable equilibrium, just a few kilometres in diameter. Free neutrons decay into protons and electrons in about ten minutes but the neutrons in a neutron star, like those comprising an atomic nucleus, do not undergo such decay. The resulting proton would have to occupy an extremely unfavourable energy state.

White dwarfs and neutron stars have been observed by radio, $X$ - and gammaray astronomers and rapidly rotating neutron stars provide an explanation for the 'pulsar' phenomenon.

The fate of the heaviest stars is well known to the average reader: unstoppable collapse to a 'black hole' state of everincreasing density. A point of no return, the 'event horizon', is encountered by infalling material and no known forces can support the star once it has been encompassed by this surface. The collapse pro- ceeds inevitably to an unknown singular state.

This is the exotic life-cycle of stellar bodies and its documentation often requires a description of matter in gravitational fields too strong for Newton's classical theory to be accurate. And this is the world that the Sexls equip the student to explore. White Dwarfs, Black Holes is a translation of a new edition of their successful German text explaining the nature of general relativity, gravitational waves, relativistic astrophysics and elementary cosmology. It is clear and concise, didactic in style and eminently suitable for finalyear undergraduates in physics or astronomy. General relativity is described in qualitative terms (no prior familiarity is assumed and no tensor analysis is developed in the text) with a good description of its experimental status although, unfortunately, the observations of the binary pulsar are not included. The authors maintain a good balance between theory and observation by concentrating upon hard, testable results rather than speculative possibilities. However, it is odd to see some of the theory of primordial nucleosynthesis developed without a glimpse at the observational data on cosmic abundances of very light elements. The authors use physical reasoning wherever possible and avoid the use of inessential mathematics. SI units are used throughout and illustrations are skilfully employed to augment, rather than merely re-state, the textual material. A series of exercises are scattered through the text and many have 'solutions' provided. Some of these questions are a little too open-ended to be useful for private study because specific background references are not provided.

Unfortunately referencing is often scanty and imprecise. There is a considerable amount of name-dropping in the text without reference to sources or follow-up articles, and the bibliography is categorized in a fashion that may be too general for students. A list of specific semi-popular articles would have been useful here. But these are second-order problems in a book that is clearly written, well produced and realistically priced. I recommend it to any student who is contemplating graduate work in astrophysics, to teachers seeking a well-ordered foundation course, or indeed to anyone curious to know what relativistic astrophysics is about.

J.D. Barrow is a Research Lecturer in the Department of Astrophysics at the University of Oxford, UK.

\section{Experts on vacua}

\section{John Yarwood}

Methods of Experimental Physics. Volume 14, Vacuum Physics and Technology. Edited by G.L. Weissler and R.W. Carlson. Pp. 593. (Academic: New York and London, 1979.) \$55, £13.80.

THIS BOOK contains a great deal of useful information, concisely and well-written by a number of American authors, all of whom are widely experienced in vacuum science and technology and have up-todate knowledge gained in academic or industrial research.

The difficulty in judging the book is to assess at whom it is aimed. Despite the fact that the purpose (according to the title of this series) is experimental, a fairly sophisticated mathematical notation is almost always used by the authors so that a reader of that useful type of person - the technician - is, by and large, ruled out. The style is really more suited to the postgraduate student of science or technology.

Roughly the first half of the text is concerned with the production and measurement of vacuum (mostly high and ultrahigh). Although the authors frequently give ideas and explanations which are novel, the topics concerned have received much attention in textbooks and journals over the past 30 years or so. One wonders, therefore, whether it wouldn't have been preferable to omit most of the well-worn material and concentrate on the more novel second half, beginning with "Metal Vacuum Systems and Components"'. Here, modern information is provided about components and materials and their most effective use in the design of vacuum systems for specific applications.

The book contains no information about how the undergraduate student should go about undertaking practical work in vacuum science and technology.

\section{First of a series on inflammation}

\section{G. P. Lewis}

Handbook of Inflammation. Volume 1, Chemical Messengers of the Inflammatory Process. Edited by J. C. Houck. Pp.422. (Elsevier/North-Holland: Amsterdam and New York, 1979.) \$75, Dfl. 154.

IN THE introduction to this first volume of the Handbook of Inflammation, the editor, John C. Houck, gives a splendid though brief account of the various steps in the inflammatory process. The book has then been arranged so that the mediators connected with each stage have been discussed.

Our old friend histamine comes first, but brought up to date in a very interesting way. In addition to the work of recent years on $\mathrm{H}_{2}$ receptor-mediated actions, the
However, it will be most useful to the member of academic staff who is concerned with the development of such practical experiments and wishes to write them up satisfactorily for undergraduates, students beginning research and for special short courses.

John Yarwood retired in 1978 as Head of Physics at the Polytechnic of Central London, $U K$, and is the editor of Vacuum.

author reviews current ideas on the possible regulatory function of histamine on activities of inflammatory cells. This theme of cell-cell interactions mediated by soluble mediators is taken up again later in the book in several chapters. Lymphokines, which were originally thought only to be the soluble products of activated T-lymphocytes, have now been shown to be not strictly restricted to immune activation pathways. The author of this particular chapter points out that release of soluble intracellular mediators might be a basic method of biological communication and describes lymphokines and cytokines as a plethora of inflammatory mediators which even includes viral and immune interferon.

The kinin system is described in some detail with the emphasis on biochemical aspects; only two of the 46 pages cover the role of kinins in inflammation. The review very adequately covers the relationships between the kinin and fibrinolytic systems and Hagemann factor, and the repetition 